\title{
Lipoma of the Parotid Gland: A Case Report
}

\author{
Bouatay $\mathbf{R}^{1,2 *}$, Kolsi $\mathbf{N}^{1,2}$, Bouaziz $\mathbf{N}^{1,2}$, Harrathi $\mathbf{k}^{1,2}$ and Koubaa J,2 \\ ${ }^{1}$ Department of Otorhinolaryngology, Head and Neck Surgery at "Fattouma Bourguiba" Hospital, Tunisia \\ ${ }^{2}$ University of Monastir, Tunisia
}

*Corresponding author: Bouatay Rachida, Department of Otorhinolaryngology, Head and Neck Surgery at "Fattouma Bourguiba"

Hospital, Monastir, University of Monastir, Tunisia

\begin{abstract}
Lipoma of the parotid gland is extremely rare, accounting for only $0,6 \%-4,4 \%$ of all parotid tumors. We present a rare case of lipoma of the superficial parotid lobe. A 68 - year-old man, presented in our department with a mass of the left parotid region. Clinical examination revealed a mobile, soft, non-tender mass in the area of the left parotid gland. MRI concluded to a lipoma of the superficial lobe of parotid gland. Parotidectomy preserving the facial nerve was performed. No complication nor recurrence were noted after a follow-up of 12 months. Lipoma arising in the parotid gland is extremely rare. Resection of this tumor requires full exposure of the facial nerve and its branches.
\end{abstract}

Keywords: Lipoma; Parotid gland; Superficial lobe; Parotidectomy

\section{Introduction}

Lipoma is a common mesenchymal soft tissue tumor that can be found in any part of the body. It can develop in the head and neck region in $15-20 \%$ of cases. Rarely it can arise in the Parotid gland with a ranging incidence from 0,6 to $4,4 \%$ [1]. Clinical diagnosis may be difficult. MRI is necessary, in diagnosis [2]. Surgical management of these tumors is challenging and need meticulous dissection of the facial nerve. We report a case of lipoma arising of the parotid gland and we discuss through literature its clinical and therapeutic features.

\section{Case Report}

A 68 - year-old man presented with mass of the left parotid region, which was painless slow-growing for 5 years. Clinical examination revealed a mobile, soft, non-tender mass that measured about $6 \mathrm{~cm}$ in diameter in the area of the left parotid gland, extending from the ear lobule to the left mandibular angle. There was no facial paralysis nor evidence of cervical lymphadenopathy. Magnetic resonance imaging (MRI) showed a well-defined homogeneous lesion of the superficial lobe of the left parotid gland with an enhanced signal on T1- and T2-weighted sequences and weak signal on fat suppressed sequences (Figure 1). The diagnosis of intra parotid lipoma was evoked. A left superficial parotidectomy preserving the facial nerve was performed. The specimen was soft, yellowish, well- circumscribed measuring $80 * 34 \mathrm{~mm}$. Histological examination revealed a well-circumscribed aggregate of mature adipocytes surrounded by a thin fibrous capsule confirming the diagnosis of intraparotid lipoma. No recurrence or complication were observed after a follow-up of 12 months.

\section{Discussion}

Lipoma is one of the most frequently encountered benign mesenchymal tumors that may originate from adipose tissue in any part of the body $[3,4]$. Rarely, it can develop in the parotid gland with reported incidence ranging from $0,6 \%$ to $4,4 \%$ among parotid tumors [3,5]. Lipoma may occur at any age, but most frequently between 40 and 60 years with a male predominance [5,6]. Its aetiology is unknown. It can be caused by heredity, obesity, diabetes, radiation, endocrine disorders, insulin injection, corticosteroid therapy and trauma [7]. In our case, we did not find any aetiological factors. Most of the reported cases were located at the superficial parotid lobe [7]. Lipomas involving the deep parotid lobe are extremely rare [5-8]. Clinical diagnosis may be difficult [9], especially for tumors located at the deep parotid lobe because it is difficult to evaluate the relationship between these masses and the surrounding tissues. Those situated at superficial parotid lobe usually appear as a slow growing, non-tender, movable and well- 
differentiated soft mass in parotid region [3-10]. Facial paralysis and pain are uncommon signs and rarely have been described [5-11]. This benign clinical presentation is most often mistaken for Warthin tumor or pleomorphic adenoma [3-12]. Fine needle aspiration cytology (FNAC) has great value in the diagnosis of parotid tumors and requires an experienced cytologist. Its accuracy drops to less than $50 \%$ in the cases of parotid lipomas [5-13]. On imaging, CT scan shows hypodense, homogeneous and well delineated mass with few septations and negative attenuation, without contrast enhancement [3-13]. However, CT scan cannot distinguish lipoma from surrounding adipose tissue. MRI remains the best diagnostic tool that can accurately diagnose lipomas [5-1]. Lipomas produce strong signals on T1- and T2 weighted sequences and weak signals on fat-suppressed sequences. After Gadolinium injection, the mass still hypointense to parotid, homogeneous in signal and uniformly non-enhancing [3]. MRI can also clearly define the limits of lipoma from normal adipose tissue and may be useful in determining the appropriate surgical approach.
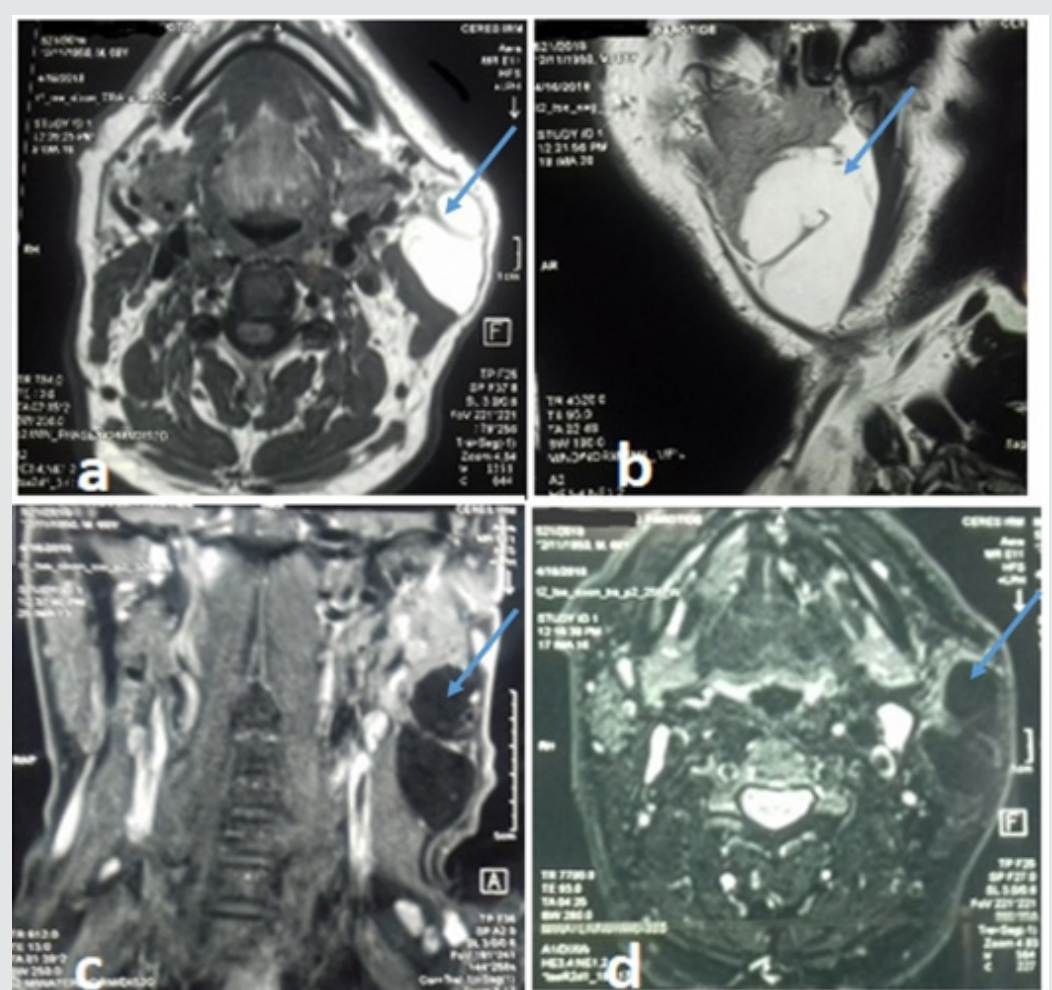

Figure 1: MRI of the parotid gland showing homogeneous mass on the superficial lobe of the left parotid gland on hyper signal $\mathrm{T} 1$ and T2 and a weak signal on fat suppressed sequences. The lesion is hypointense to parotid and uniformly non-enhancing.

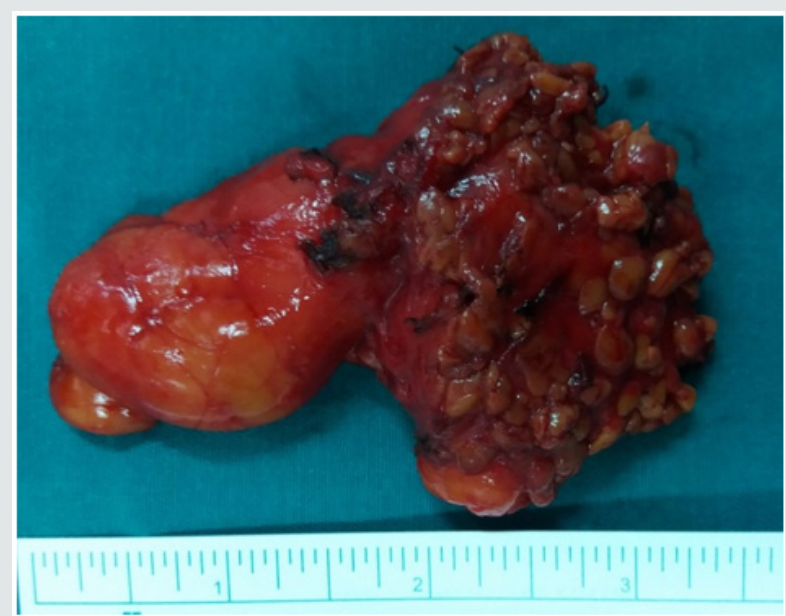

Figure 2: Macroscopic findings of the resected tumor.

Histopathologic investigation reveals mature adipose tissue separated from parotid gland parenchyma with a fibrous capsule. Identification of a capsule may aid in distinguishing such a neoplasm from pseudolipoma, lobular lipomatous atrophy, or lipomatosis, all of which are unencapsulated [3]. Surgery is the treatment of choice of parotid gland lipoma, but its modalities remain controversial [8-12]. It should be performed by experienced surgeons because of the need for meticulous dissection of the facial nerve branches. 
The postoperative esthetic and functional results should be the major concerns [1]. Some surgeons recommend simple enucleation of a superficial lobe parotid lipoma with a small border of healthy parotid gland parenchyma, as this is easy to perform because of the well-defined capsule. Other surgeons suggest that the surgical management of parotid lipoma should be the same as that for other parotid tumors [5]. However, it is well known that transient facial nerve dysfunction and Frey's syndrome may occur as complications following surgical intervention for parotid tumors and should be explained to the patient before operation [5]. Facial nerve dysfunction ranged between 8.2 and $65 \%$ after parotid gland surgery for benign tumors [10]. Therefore, it requires efforts such as facial nerve monitoring to identify the facial nerve. Recurrence rate of parotid lipoma after adequate resection is very low. It has been reported in $5 \%$ in all cases when it is well-encapsulated [59]. Malignant transformation into liposarcomas has been reported in few cases in the literature [9]. Therefore, careful follow-up is recommended.

\section{Conclusion}

Lipoma of the parotid gland is a rare benign tumor, which should be considered in the differential diagnosis of parotid gland's mass lesions. MRI is essential to locate the tumor, and to precise its relationship with the facial nerve. Their management is challenging. Definitive diagnosis can only be accomplished with histopathologic review.

\section{Refernces}

1. Debnatha SC, Saikia A (2010) Lipoma of the parotid gland extending from the superficial to the deep lobe : a rarity. Br J Oral Maxillofac Surg 48: 203-204.
2. Kimura Y, Ishikawa N, Goutsu K, Kitamura K, Kishimoto S (2002) Lipoma in the deep lobe of the parotid gland: a case report. Auris Nasus Larynx 29: 391-393

3. Kimberly NT, Seltzer S, Castle T (2019) Lipoma of the Parotid Gland. Head Neck Pathol.

4. Baykul TM, Aydin A, Findik Y, Yildirim D (2016) Huge lipoma of the right parotid gland: Case report and review of 42 cases. ENT Journal 95(1): 8-9.

5. Mohammadi G, Notash R (2014) A rare case of bilobe parotid lipoma: Involving both superficial and deep lobe of parotid. Egyptian Journal of Ear, Nose, Throat and Allied Sciences 15: 69-71.

6. Touil D, Hasni W, Mziou Z, Zaghbani A, Nouma B, et al. (2015) Adipocytic tumors of the salivary glands : A retrospective study. Rev Stomatol Chir Maxillofac Chir Oral 116: 296-301.

7. Rehal SS, Alibhai M, Perera E (2016) Lipoma of the parotid gland after trauma: a case report and review. Br J Oral Maxillo fac Surg 55: 5-6.

8. Ulku CH, Uyar Y, Unaldi D (2005) Management of lipomas arising from deep lobe of the parotid gland. Auris Nasus Larynx 32: 49-53.

9. Morita N, Todo K, Yabuta T, Ootuki R (2007) Lipoma of the Parotid Gland. Asian J Oral Maxillofac Surg 19: 107-109.

10. Tilaveridis I, Kalaitsidou I, Pastelli N, Antoniades K (2018) Lipoma of Parotid Gland: Report of Two Cases. J. Maxillofac Oral Surg 17(4): 453457.

11. Des Roziers BB, Azoulay B, Lasta S, Dumas L, Cartier S (2008) Lipoma widened to both lobes of the parotid gland: An exceptional entity. Case report. Ann Chir Plast Esthet 53: 285-288.

12. Ohyama Y, Shigematsua H, Kawamotoa Y, Takizawaa S, Kikuchi K, et al. (2012) A case of Sdeep lobe parotid lipoma. J Oral Maxillofac Surg Med Pathol 24: 132-135.

13. Harada H, Omura K (2003) Lipoma of the Parotid Gland Asian J Oral Maxillofac Surg 15: 292-294.
This work is licensed under Creative Commons Attribution 4.0 License

To Submit Your Article Click Here: Submit Article

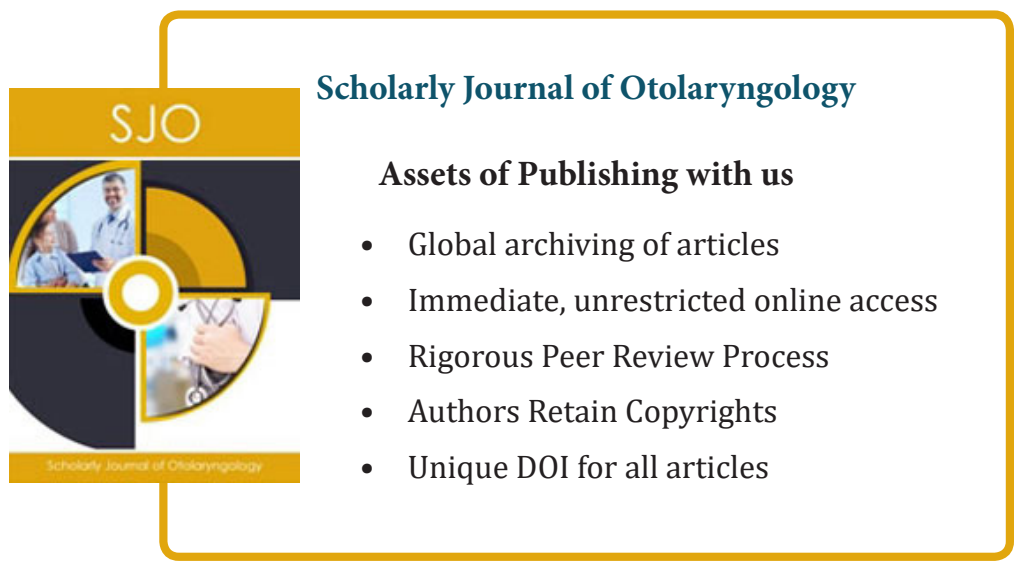

